\title{
Structural impact on SARS-CoV-2 spike protein by D614G substitution
}

\author{
Jun Zhang,,2中, Yongfei Cai ${ }^{1,2}+$, Tianshu Xiao,,2, Jianming Lu ${ }^{3}$, Hanqin Peng1, Sarah M. Sterling, ${ }^{4,5}$, Richard M. \\ Walsh Jr. ${ }^{4,5}$, Sophia Rits-Volloch', Haisun Zhu' ${ }^{6}$, Alec N. Woosley ${ }^{6}$, Wei Yang ${ }^{6}$, Piotr Sliz ${ }^{1,2,5}$, Bing Chen ${ }^{1,2 *}$
}

${ }^{1}$ Division of Molecular Medicine, Boston Children's Hospital, Boston, MA 02115, USA. ${ }^{2}$ Department of Pediatrics, Harvard Medical School, Boston, MA 02115, USA. ${ }^{3}$ Codex BioSolutions, Inc., Gaithersburg, MD 20879, USA. ${ }^{4}$ The Harvard Cryo-EM Center for Structural Biology, Harvard Medical School, Boston, MA 02115, USA. ${ }^{5}$ Department of

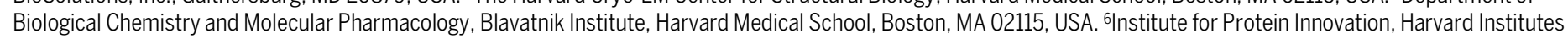
of Medicine, Boston, MA 02115, USA.

†These authors contributed equally to this work.

*Corresponding author. Email: bchen@crystal.harvard.edu

Substitution for aspartic acid by glycine at position 614 in the spike (S) protein of severe acute respiratory syndrome coronavirus 2 appears to facilitate rapid viral spread. The G614 strain and its recent variants are now the dominant circulating forms. We report here cryo-EM structures of a full-length G614 S trimer, which adopts three distinct prefusion conformations differing primarily by the position of one receptorbinding domain. A loop disordered in the D614 S trimer wedges between domains within a protomer in the G614 spike. This added interaction appears to prevent premature dissociation of the G614 trimer, effectively increasing the number of functional spikes and enhancing infectivity, and to modulate structural rearrangements for membrane fusion. These findings extend our understanding of viral entry and suggest an improved immunogen for vaccine development.

Severe acute respiratory syndrome coronavirus 2 (SARS-CoV2), an enveloped positive-stranded RNA virus, is the cause of the COVID-19 pandemic (1). Although the viral evolution is slowed by the RNA proofreading capability of its replication machinery (2), a variant with a single-residue substitution (D614G) in its spike (S) protein rapidly became the dominant strain throughout the world (3). It has since further evolved to give several variants of concern (VOCs) (4-6). The trimeric $\mathrm{S}$ protein decorates the viral surface and is an important target for development of diagnostics, therapeutics and vaccines, therefore understanding the effect of key mutations may guide intervention strategies. Here we focus on D614G mutation that is in all currently circulating strains. The S protein is produced as a single-chain precursor and subsequently processed by a furin-like protease into the receptor-binding fragment S1 and the fusion fragment S2 (7). After engagement of the receptor-binding domain (RBD) in S1 with the viral receptor angiotensin converting enzyme 2 (ACE2) on the host cell surface, followed by a second proteolytic cleavage within S2 (S2' site) (8), the S protein undergoes large conformational changes, resulting in dissociation of $\mathrm{S} 1$ and irreversible refolding of S2 into a postfusion structure $(9,10)$. This induces fusion of the virus and host cell membranes to initiate infection. Rapid advances in structural biology of the SARS-CoV-2 $\mathrm{S}$ protein include structures of its soluble fragments: the ectodomain stabilized in its prefusion conformation (11-13),
RBD-ACE2 complexes (14-17), and segments of S2 in the postfusion state (18). In the prefusion ectodomain structure, S1 folds into four domains - NTD (N-terminal domain), RBD, and two CTDs (C-terminal domains), and wraps around the prefusion S2, with the RBD sampling two distinct conformations - "up" for a receptor-accessible state and "down" for a receptor-inaccessible state. We and others have reported structures of a purified, full-length $\mathrm{D} 614$ S protein in both prefusion and postfusion conformations $(19,20)$. Studies by cryo-electron tomography, with chemically inactivated SARSCoV-2 preparations, using both D614 and G614 variants have revealed additional structural details of $S$ proteins present on the surface of virion (21-24).

Epidemiological surveillance indicated that the SARSCoV-2 carrying G614 outcompeted the original virus and became the globally dominant form within a month $(3,25,26)$. This single-residue substitution appears to correlate with high viral loads in infected patients and high infectivity of pseudotyped viruses, but not with disease severity (3). The G614 virus has comparable sensitivity to neutralization by convalescent human sera or vaccinated hamster sera $(3,27-$ 30 ), suggesting that vaccines containing D614 remain effective against the G614 virus. Moreover, S1 dissociates more readily from the D614 virus than from G614 virus (31), indicating that the D614 viral spike is less stable than the G614 variant. The G614 ectodomain trimer is reported to sample 
the RBD-up conformations more frequently than does the D614 trimer $(13,29,32)$, but it is puzzling why the former binds more weakly to recombinant ACE2 than the latter (32). The known $\mathrm{S}$ trimer structures indicate that the D614G change breaks a salt bridge between D614 and a lysine residue (K854) in the fusion peptide proximal region (FPPR) (19, $33,34)$, which may help clamp the RBD in the prefusion conformation. This observation can explain why the G614 trimer favors the RBD-up conformations, but does not account for its increased stability. To resolve these issues, we report here the structural consequences of the D614G substitution in the context of the full-length $\mathrm{S}$ protein.

We compared the membrane fusion activity of the fulllength G614 S protein (fig. S1) with that of the D614 S construct in a cell-cell fusion assay (19). All the cells expressing $\mathrm{S}$ fused efficiently with cells transfected with a human ACE2 construct (fig. S2A), demonstrating that the S proteins expressed on the cell surfaces are fully functional. At low transfection levels, G614 S had higher fusion activity than the D614 $\mathrm{S}$, but the difference diminished with the increased amount of transfected DNA, suggesting that the high expression levels can compensate for lower fusion efficiency of the D614 S protein. The G614 trimer remains sensitive to inhibition by an engineered trimeric ACE2-based inhibitor that competes with the receptor on the target cells (35) (fig. S2B). For protein purification, we used a construct fused with a C-terminal strep-tag, which was equally active in cell-cell fusion as the untagged version (fig. S2A), and purified both G614 and D614 proteins under identical conditions. The D614 protein eluted in three peaks characterized previously as the prefusion $\mathrm{S}$ trimer, the postfusion $\mathrm{S} 2$ trimer and the dissociated monomeric S1 (19). The G614 protein eluted as a single major peak, corresponding to the prefusion trimer (Fig. 1A). This suggests that D614G has a striking effect on the stability of the SARSCoV-2 S trimer. Coomassie-stained SDS-PAGE analysis shows that G614 elutes mainly as the prefusion trimer, comprising the cleaved S1/S2 complex ( 90\%) and a small amount of the uncleaved S precursor ( $10 \%)$. We next measured binding of the prefusion trimer fractions of the full-length proteins to recombinant soluble ACE2 by bio-layer interferometry (BLI) (Fig. 1B). The $\mathrm{S}$ trimers bound more strongly to a dimeric ACE2 than to a monomeric ACE2, as expected. The G614 protein bound ACE2 less tightly than did the D614 protein, consistent with the measurements reported by others using soluble constructs (32). This observation appears inconsistent with reports that the G614 trimer has a more exposed RBD than the D614 trimer $(13,21,22,29,32)$. We note that the second binding event between dimeric ACE2 and a G614 trimer has both a slower on-rate and a slower off-rate than for a D614 trimer (table S1).

We determined the cryo-EM structures of the full-length G614 S trimer using RELION (36). 3D classification identified three distinct classes each containing a similar number of particles. The three classes represent a closed, three RBDdown conformation, a one RBD-up conformation and an intermediate conformation with one RBD flipped up only halfway. All structures were refined to 3.1-3.5 $\AA$ resolution (figs. S3 to S8 and table S2). The overall structure of the G614 S protein in the closed, three RBD-down prefusion conformation is very similar to that of our published D614 S trimer (Fig. 2) (19). In the three RBD-down structure, the four domains in each S1, including NTD, RBD, CTD1 and CTD2, wrap around the three-fold axis of the trimer, protecting the prefusion S2. The furin cleavage site is disordered, making it uncertain whether this structure represents the uncleaved or cleaved trimer, although the preparation contains primarily the cleaved forms (Fig. 1A). The S2 fragment folds around a central three-stranded coiled coil that forms the most stable part of the structure; it is also the least variable region among all the known $\mathrm{S}$ trimer structures. The S2 structure is identical in the two G614 structures with one RBD projecting upwards, either completely or partially (fig. S9). In the conformation with one RBD fully up, the two neighboring NTDs, including the one from the same protomer, shift away from the three-fold axis (fig. S9). In the RBD intermediate conformation, only the NTD from the adjacent protomer packing directly against the moving RBD shifts. The D614G substitution eliminates a salt bridge between D614 in CTD2 of one subunit and K854 in the FPPR of the adjacent subunit $(19,34)$, but the FPPR in the three RBD-down conformation of the G614 trimer remains structured.

To examine the structural changes resulted from the D614G substitution, we superposed the structures of the G614 trimer onto the D614 trimer in the closed conformation aligning them by the invariant S2 (Fig. 2B). A shift by a clockwise, outward rotation of all three S1 subunits, relative to the D614 structure, is evident even for the G614 trimer in the closed conformation. A similar shift was also observed in the RBDintermediate and RBD-up G614 structures. Thus, the D614G substitution has led to a slightly more open conformation than that of the D614 trimer, even when all three RBDs are down. The D614G change has apparently also rigidified a neighboring segment of CTD2, residues 620-640, which we designate the "630 loop". This loop inserts into a gap, slightly wider in the G614 than in the D614 trimer, between the NTD and CTD1 of the same protomer (Figs. 3 and 4). The 630 loop is disordered in the closed D614 trimer (fig. S10), because the gap is too narrow for it to insert. The closed D614 trimer thus has three ordered FPPRs and three disordered 630 loops, while the closed G614 trimer has three structured 630 loops along with three ordered FPPRs. In the two conformers with one partly or fully open $\mathrm{RBD}$, the two segments are disordered in the RBD-shifted subunit, and their central parts have difficult-to-model density in one other subunit. The 
third pair appears well ordered throughout (Fig. 3). Thus, opening of the RBD in the full-length G614 trimer correlates with a displacement of the 630 loop, and the FPPR away from its position in the D614 trimer. The D614G change did not cause any large local structural rearrangements except for loss of the D614-K854 salt bridge (19, 34), and a small shift of residue 614 toward the three-fold axis (Fig. 4A). The position of the FPPR and the conformation of K854 may allow a hydrogen bond between the K854 amino group and the mainchain carbonyl of G614, perhaps accounting for the subtlety of the structural difference (fig. S11, A and B). Although the loss of the salt bridge involving D614 does not destabilize the packing of the FPPR against the rest of the trimer, it does weaken the FPPR density, especially between residues 842846. The 630 loop, which packs directly against the NTD, CTD1 and CTD2 of the same protomer, lies close to the S1/S2 boundary of the same protomer and the FPPR of an adjacent protomer (Fig. 4B). Inserting this wedge-like loop between the NTD and CTD1 (Fig. 4C) may help secure the positions of the NTD and CTDs. CTD2 is formed by two stacked, fourstrand $\beta$-sheets, with a fifth strand in one sheet contributed by the connector between the NTD and RBD. In the other sheet, an interstrand loop contains the S1/S2 cleavage site, and thus one strand is the N-terminal segment of S2 (Fig. 4B). In the G614 trimer, one side of the 630 loop packs along a long hydrophobic surface, largely solvent-exposed in the D614 trimer, formed by residues on the "upward" facing surface of the CTD2 along with Pro295 from the NTD (Fig. 4D). Trp633 and Tyr636 of the 630 loop appear to contribute to this interaction. $\mathrm{S} 1$ dissociation from $\mathrm{S} 2$ requires breaking the S2 strand from the second $\beta$-sheet. An ordered 630 loop that stabilizes the CTD2 by closing off an exposed, hydrophobic surface may retard S1 shedding, thereby enhancing the stability of a cleaved $\mathrm{S}$ trimer. We note that the density for a fatty acid ligand making contacts with the neighboring RBDs in the D614 trimer is absent in all the G614 reconstructions (fig. S11C) (37), suggesting that the ligand is not required for three RBDs to adopt the down conformation. To test the impact of the 630 loop on S1 shedding and membrane fusion, we generated five $S$ mutants each containing a single residue change either in the 630 loop (W633A, R634E, V635K and Y636A) or the CTD2 hydrophobic surface (V610K) in the G614 sequence. These mutants expressed the same level of $\mathrm{S}$, with a similar extent of cleavage between $\mathrm{S} 1$ and $\mathrm{S} 2$, as expected (fig. S12A). When detected by monoclonal antibodies using flow cytometry, mutants V610K and W633A showed markedly lower binding of RBD-specific antibodies [REGN10933 and REGN10987; (38)], and of an NTD-specific antibody [4A8; (39)] than the parental G614 S, while binding to an S2specific antibody [0304-3H3; (39)] was slightly higher (fig. $\mathrm{S} 12 \mathrm{~B})$. These results are consistent with the hydrophobic interactions between the 630 loop and CTD2 stabilizing the cleaved S1/S2 complex and preventing S1 dissociation. The mutant $\mathrm{V} 635 \mathrm{~K}$ had wildtype phenotypes in these assays, likely because V635 does not make any direct contact with the CTD2. The mutants R634E and Y636A showed intermediate levels of antibody binding because Y636 appears to contribute less to the 630 loop-CTD2 interaction than W633 and R634 may help maintain the loop's overall shape for inserting between domains. Likewise, a similar pattern was observed with these mutants in the cell-cell fusion assay except that Y636A showed substantially weaker fusion activity than R634E (fig. S12C). Thus key residues important for stabilizing the $\mathrm{S}$ trimer structure appear critical for membrane fusion activity, as premature dissociation of S1 would lead to inactivation of the $\mathrm{S}$ trimer. To further confirm the folding of the 630 loop in the G614 trimer, we collected additional data under slightly different conditions and found the same three classes representing the closed, RBD-intermediate and one RBD-up conformations (fig. S13A). There is relatively strong density in both $2 \mathrm{D}$ class averages and $3 \mathrm{D}$ reconstructions for the heptad repeat 2 (HR2) region (fig. S1) and detergent micelle, invisible in all our previous cryo-EM analysis. The increased length and lack of symmetry limited the resolution of these $3 \mathrm{D}$ reconstructions to $4.3-4.7 \AA$. Nevertheless, the density for the 630 loop was evident in the closed trimer even at this resolution (fig. S13B). We note that although S1 in the G614 trimer moves outwards from its position in the D614 trimer, the extent of the shift is still appreciably smaller than the shift seen in soluble $\mathrm{S}$ trimers stabilized by a trimerization foldon tag and two proline mutations (fig. S14).

Our structures provide an explanation for why the G614 virus, with a more stable $S$ trimer, is more infectious than the original strain (fig. S15). The transition from the closed to one RBD-up conformation in a G614 trimer requires an order-disorder transition in one 630 loop and partial disordering of a second. Thus, kinetic barriers will probably make both the forward and reverse transitions slower than in a D614 trimer, in which all three 630 loops are unstructured in both conformations. In the one RBD-up conformation, $\mathrm{S}$ will also shed S1 much more slowly from a G614 trimer than from D614, because the remaining two RBDs are stabilized by the ordered and partially ordered 630 loops, and a return of the first RBD to the down configuration can occur unless locked in place by ACE2 receptor binding. This picture can account both for the greater prevalence of a one-up RBD conformation and a lower overall ACE2 affinity, because the other two RBDs will remain inaccessible. It can also explain why we captured very few trimers in the RBD-up conformation in our previous cryo-EM study of the D614 trimer but instead saw abundant postfusion S2 (19), because any one RBD-up conformation would proceed to two RBD-up and three RBD-up and shed very quickly, allowing $\mathrm{S} 2$ to convert to the postfusion form. Our interpretation of the structural differences is also 
consistent with the spike conformational distribution on the virions in cryo-ET studies of chemically inactivated SARSCoV-2. The D614 preparation contains primarily postfusion S2 spikes (24). One study of a G614 virus that had lost the furin cleavage site showed almost no postfusion spikes, and a 50:50 distribution of prefusion spikes between fully closed and one RBD-up (21); and another showed 3\% postfusion spikes and $97 \%$ in the prefusion form ( $31 \%$, fully closed; $\sim 55 \%$, one RBD-up; $\sim 14 \%$ two RBD-up) (22). The structured 630 loop in the G614 trimer not only reinforces the packing among three protomers, but also stabilizes the CTD2 to inhibit release of the N-terminal segment of S2, effectively blocking S1 dissociation. This property can account for the paucity of postfusion spikes on the G614 variant. In addition to the FPPR that might modulate the fusogenic structural rearrangements of S protein (19), CTD2 and the 630 loop within it, are probably also the key components of the S fusion machinery. If ACE2 captures the RBD-up conformation (40), expelling both the 630 loop and the FPPR from their positions in the closed S trimer conformation, the FPPR shift may help expose the S2' site near the fusion peptide for proteolytic cleavage, while departure of the 630 loop from the hydrophobic surface of the CTD2 can destabilize this domain and free the N-terminal segment of S2 to dissociate from S1, if the furin site has already been cleaved, and release $\mathrm{S} 1$ altogether. Dissociation of S1 would then initiate a cascade of refolding events in the metastable prefusion S2, allowing the fusogenic transition to a stable postfusion structure. This model is similar to that proposed for membrane fusion catalyzed by HIV envelope protein (41). The SARS-CoV-2 S protein is the centerpiece of the first-generation vaccines that almost all used the D614 sequence. The G614 S trimer is naturally constrained in a prefusion state that presents both the RBDdown and RBD-up conformations with great stability. It is therefore likely to be a superior immunogen for eliciting protective neutralizing antibody responses, which appear largely to target the RBD and NTD $(39,42)$. It may also be an excellent scaffold for designing next-generation vaccines against new variants that have become resistant to protections offered by the existing vaccines (43-46). In summary, we suggest that the enhanced infectivity of the G614 virus largely results from the increased stability of the $S$ trimer, rather than the better exposed RBDs. Indeed, if the virus that passed from bats to humans or to an intermediate vector contained D614 [also present in the bat coronavirus BatCoV RaTG13 (1)], then it could have gained fitness in the new host by acquiring changes such as G614 for greater stability and infectivity than the parental form. Not surprisingly, the recent fast-spreading variants, including the B.1.1.7 (VUI202012/01; 501Y.V1) lineage from the United Kingdom, the B.1.351 (501Y.V2) lineage from South Africa and the B.1.1.28 (484K.V2; P.1) lineage from Brazil (4-6), all contain the
D614G substitution (table S3), suggesting that the increased transmissibility of the G614 virus has led to a great number of replication events and to greater genetic diversity, despite a lower absolute mutation rate.

\section{REFERENCES AND NOTES}

1. P. Zhou, X.-L. Yang, X.-G. Wang, B. Hu, L. Zhang, W. Zhang, H.-R. Si, Y. Zhu, B. Li, C.L. Huang, H.-D. Chen, J. Chen, Y. Luo, H. Guo, R.-D. Jiang, M.-Q. Liu, Y. Chen, X.-R. Shen, X. Wang, X.-S. Zheng, K. Zhao, Q.-J. Chen, F. Deng, L.-L. Liu, B. Yan, F.-X. Zhan, Y.-Y. Wang, G.-F. Xiao, Z.-L. Shi, A pneumonia outbreak associated with a new coronavirus of probable bat origin. Nature 579, 270-273 (2020). doi:10.1038/s41586-020-2012-7 Medline

2. F. Robson, K. S. Khan, T. K. Le, C. Paris, S. Demirbag, P. Barfuss, P. Rocchi, W.-L. $\mathrm{Ng}$, Coronavirus RNA Proofreading: Molecular Basis and Therapeutic Targeting. Mol. Cell 79, 710-727 (2020). doi:10.1016/j.molcel.2020.07.027 Medline

3. B. Korber, W. M. Fischer, S. Gnanakaran, H. Yoon, J. Theiler, W. Abfalterer, N. Hengartner, E. E. Giorgi, T. Bhattacharya, B. Foley, K. M. Hastie, M. D. Parker, D. G. Partridge, C. M. Evans, T. M. Freeman, T. I. de Silva, Sheffield COVID-19 Genomics Group, C. McDanal, L. G. Perez, H. Tang, A. Moon-Walker, S. P. Whelan, C. C. LaBranche, E. O. Saphire, D. C. Montefiori, Tracking Changes in SARS-CoV2 Spike: Evidence that D614G Increases Infectivity of the COVID-19 Virus. Cell 182, 812-827.e19 (2020). doi:10.1016/j.cell.2020.06.043 Medline

4. H. Tegally, E. Wilkinson, M. Giovanetti, A. Iranzadeh, V. Fonseca, J. Giandhari, D. Doolabh, S. Pillay, E. J. San, N. Msomi, K. Mlisana, A. von Gottberg, S. Walaza, M. Allam, A. Ismail, T. Mohale, A. J. Glass, S. Engelbrecht, G. Van Zyl, W. Preiser, F. Petruccione, A. Sigal, D. Hardie, G. Marais, M. Hsiao, S. Korsman, M.-A. Davies, L. Tyers, I. Mudau, D. York, C. Maslo, D. Goedhals, S. Abrahams, O. Laguda-Akingba, A. Alisoltani-Dehkordi, A. Godzik, C. K. Wibmer, B. T. Sewell, J. Lourenço, L. C. J. Alcantara, S. L. Kosakovsky Pond, S. Weaver, D. Martin, R. J. Lessells, J. N. Bhiman, C. Williamson, T. de Oliveira, Emergence and rapid spread of a new severe acute respiratory syndrome-related coronavirus 2 (SARS-CoV-2) lineage with multiple spike mutations in South Africa. medRxiv 2020.12.21.20248640 [Preprint]. 22 December 2020. https://doi.org/10.1101/2020.12.21.20248640.

5. F. Grabowski, G. Preibisch, S. Giziński, M. Kochańczyk, T. Lipniacki, SARS-CoV-2 Variant of Concern 202012/01 has about twofold replicative advantage and acquires concerning mutations. medRxiv 2020.12.28.20248906 [Preprint]. 21 February 2021. https://doi.org/10.1101/2020.12.28.20248906.

6. C. M. Voloch, R. da Silva F Jr., L. G. P. de Almeida, C. C. Cardoso, O. J. Brustolini, A. L. Gerber, A. P. de C Guimarães, D. Mariani, R. M. da Costa, O. C. Ferreira Jr., Covid19-UFR J Workgroup, LNCC-Workgroup, A. C. Cavalcanti, T. S. Frauches, C. M. B. de Mello, R. M. Galliez, D. S. Faffe, T. M. P. P. Castiñeiras, A. Tanuri, A. T. R. de Vasconcelos, Genomic characterization of a novel SARS-CoV-2 lineage from Rio de Janeiro, Brazil. medRxiv 2020.12.23.20248598 [Preprint]. 26 December 2020. https://doi.org/10.1101/2020.12.23.20248598.

7. B. J. Bosch, R. van der Zee, C. A. de Haan, P. J. Rottier, The coronavirus spike protein is a class I virus fusion protein: Structural and functional characterization of the fusion core complex. J. Virol. 77, 8801-8811 (2003). doi:10.1128/JVl.77.16.88018811.2003 Medline

8. M. Hoffmann, H. Kleine-Weber, S. Schroeder, N. Krüger, T. Herrler, S. Erichsen, T. S. Schiergens, G. Herrler, N.-H. Wu, A. Nitsche, M. A. Müller, C. Drosten, S. Pöhlmann, SARS-CoV-2 Cell Entry Depends on ACE2 and TMPRSS2 and Is Blocked by a Clinically Proven Protease Inhibitor. Cell 181, 271-280.e8 (2020). doi:10.1016/j.cell.2020.02.052 Medline

9. J. K. Millet, G. R. Whittaker, Host cell entry of Middle East respiratory syndrome coronavirus after two-step, furin-mediated activation of the spike protein. Proc. Natl. Acad. Sci. U.S.A. 111, 15214-15219 (2014). doi:10.1073/pnas.1407087111 Medline

10. M. A. Tortorici, D. Veesler, Structural insights into coronavirus entry. Adv. Virus Res. 105, 93-116 (2019). doi:10.1016/bs. aivir.2019.08.002 Medline

11. D. Wrapp, N. Wang, K. S. Corbett, J. A. Goldsmith, C.-L. Hsieh, O. Abiona, B. S. Graham, J. S. McLellan, Cryo-EM structure of the 2019-nCoV spike in the prefusion conformation. Science 367, 1260-1263 (2020). doi:10.1126/science. abb2507 Medline

12. A. C. Walls, Y.-J. Park, M. A. Tortorici, A. Wall, A. T. McGuire, D. Veesler, Structure, function, and antigenicity of the SARS-CoV-2 spike glycoprotein. Cell 181, 281- 
292.e6 (2020). doi:10.1016/i.cell.2020.02.058 Medline

13. S. M. Gobeil, K. Janowska, S. McDowell, K. Mansouri, R. Parks, K. Manne, V. Stalls, M. F. Kopp, R. Henderson, R. J. Edwards, B. F. Haynes, P. Acharya, D614G Mutation Alters SARS-CoV-2 Spike Conformation and Enhances Protease Cleavage at the S1/S2 Junction. Cell Rep. 34, 108630 (2021). doi:10.1016/i.celrep.2020.108630 Medline

14. J. Lan, J. Ge, J. Yu, S. Shan, H. Zhou, S. Fan, Q. Zhang, X. Shi, Q. Wang, L. Zhang, X. Wang, Structure of the SARS-CoV-2 spike receptor-binding domain bound to the ACE2 receptor. Nature 581, 215-220 (2020). doi:10.1038/s41586-020-2180-5 Medline

15. R. Yan, Y. Zhang, Y. Li, L. Xia, Y. Guo, Q. Zhou, Structural basis for the recognition of SARS-CoV-2 by full-length human ACE2. Science 367, 1444-1448 (2020). doi:10.1126/science.abb2762 Medline

16. J. Shang, G. Ye, K. Shi, Y. Wan, C. Luo, H. Aihara, Q. Geng, A. Auerbach, F. Li, Structural basis of receptor recognition by SARS-CoV-2. Nature 581, 221-224 (2020). doi:10.1038/s41586-020-2179-y Medline

17. Q. Wang, Y. Zhang, L. Wu, S. Niu, C. Song, Z. Zhang, G. Lu, C. Qiao, Y. Hu, K.-Y. Yuen, Q. Wang, H. Zhou, J. Yan, J. Qi, Structural and Functional Basis of SARS-CoV-2 Entry by Using Human ACE2. Cell 181, 894-904.e9 (2020). doi:10.1016/i.cell.2020.03.045 Medline

18. S. Xia, M. Liu, C. Wang, W. Xu, Q. Lan, S. Feng, F. Qi, L. Bao, L. Du, S. Liu, C. Qin, F. Sun, Z. Shi, Y. Zhu, S. Jiang, L. Lu, Inhibition of SARS-CoV-2 (previously 2019$\mathrm{nCoV}$ ) infection by a highly potent pan-coronavirus fusion inhibitor targeting its spike protein that harbors a high capacity to mediate membrane fusion. Cell Res. 30, 343-355 (2020). doi:10.1038/s41422-020-0305-x Medline

19. Y. Cai, J. Zhang, T. Xiao, H. Peng, S. M. Sterling, R. M. Walsh Jr., S. Rawson, S. RitsVolloch, B. Chen, Distinct conformational states of SARS-CoV-2 spike protein. Science 369, 1586-1592 (2020). doi:10.1126/science.abd4251 Medline

20. S. Bangaru, G. Ozorowski, H. L. Turner, A. Antanasijevic, D. Huang, X. Wang, J. L. Torres, J. K. Diedrich, J.-H. Tian, A. D. Portnoff, N. Patel, M. J. Massare, J. R. Yates 3rd, D. Nemazee, J. C. Paulson, G. Glenn, G. Smith, A. B. Ward, Structural analysis of full-length SARS-CoV-2 spike protein from an advanced vaccine candidate. Science 370, 1089-1094 (2020). doi:10.1126/science.abe1502 Medline

21. B. Turoňová, M. Sikora, C. Schürmann, W. J. H. Hagen, S. Welsch, F. E. C. Blanc, S. von Bülow, M. Gecht, K. Bagola, C. Hörner, G. van Zandbergen, J. Landry, N. T. D. de Azevedo, S. Mosalaganti, A. Schwarz, R. Covino, M. D. Mühlebach, G. Hummer, J. Krijnse Locker, M. Beck, In situ structural analysis of SARS-CoV-2 spike reveals flexibility mediated by three hinges. Science 370, 203-208 (2020). doi:10.1126/science.abd5223 Medline

22. Z. Ke, J. Oton, K. Qu, M. Cortese, V. Zila, L. McKeane, T. Nakane, J. Zivanov, C. J. Neufeldt, B. Cerikan, J. M. Lu, J. Peukes, X. Xiong, H.-G. Kräusslich, S. H. W. Scheres, R. Bartenschlager, J. A. G. Briggs, Structures and distributions of SARSCoV-2 spike proteins on intact virions. Nature 588, 498-502 (2020). doi:10.1038/s41586-020-2665-2 Medline

23. H. Yao, Y. Song, Y. Chen, N. Wu, J. Xu, C. Sun, J. Zhang, T. Weng, Z. Zhang, Z. Wu, L. Cheng, D. Shi, X. Lu, J. Lei, M. Crispin, Y. Shi, L. Li, S. Li, Molecular Architecture of the SARS-CoV-2 Virus. Cell 183, 730-738.e13 (2020). doi:10.1016/i.cell.2020.09.018

24. C. Liu, C. Liu, L. Mendonça, Y. Yang, Y. Gao, C. Shen, J. Liu, T. Ni, B. Ju, C. Liu, X. Tang, J. Wei, X. Ma, Y. Zhu, W. Liu, S. Xu, Y. Liu, J. Yuan, J. Wu, Z. Liu, Z. Zhang, L. Liu, P. Wang, P. Zhang, The Architecture of Inactivated SARS-CoV-2 with Postfusion Spikes Revealed by Cryo-EM and Cryo-ET. Structure 28, 1218-1224.e4 (2020). doi:10.1016/i.str.2020.10.001 Medline

25. Q. Li, J. Wu, J. Nie, L. Zhang, H. Hao, S. Liu, C. Zhao, Q. Zhang, H. Liu, L. Nie, H. Qin, M. Wang, Q. Lu, X. Li, Q. Sun, J. Liu, L. Zhang, X. Li, W. Huang, Y. Wang, The Impact of Mutations in SARS-CoV-2 Spike on Viral Infectivity and Antigenicity. Cell 182, 1284-1294.e9 (2020). doi:10.1016/i.cell.2020.07.012 Medline

26. S. Isabel, L. Graña-Miraglia, J. M. Gutierrez, C. Bundalovic-Torma, H. E. Groves, M. R. Isabel, A. Eshaghi, S. N. Patel, J. B. Gubbay, T. Poutanen, D. S. Guttman, S. M. Poutanen, Evolutionary and structural analyses of SARS-CoV-2 D614G spike protein mutation now documented worldwide. Sci. Rep. 10, 14031 (2020). doi:10.1038/s41598-020-70827-z Medline

27. J. A. Plante, Y. Liu, J. Liu, H. Xia, B. A. Johnson, K. G. Lokugamage, X. Zhang, A. E. Muruato, J. Zou, C. R. Fontes-Garfias, D. Mirchandani, D. Scharton, J. P. Bilello, Z. Ku, Z. An, B. Kalveram, A. N. Freiberg, V. D. Menachery, X. Xie, K. S. Plante, S. C.
Weaver, P.-Y. Shi, Spike mutation D614G alters SARS-CoV-2 fitness. Nature 10.1038/s41586-020-2895-3 (2020). doi:10.1038/s41586-020-2895-3 Medline 28. J. Hu, C.-L. He, Q.-Z. Gao, G.-J. Zhang, X.-X. Cao, Q.-X. Long, H.-J. Deng, L.-Y. Huang, J. Chen, K. Wang, N. Tang, A.-L. Huang, D614G mutation of SARS-CoV-2 spike protein enhances viral infectivity. bioRxiv 2020.06.20.161323 [Preprint]. 6 July 2020. https://doi.org/10.1101/2020.06.20.161323.

29. D. Weissman, M.-G. Alameh, T. de Silva, P. Collini, H. Hornsby, R. Brown, C. C. LaBranche, R. J. Edwards, L. Sutherland, S. Santra, K. Mansouri, S. Gobeil, C. McDanal, N. Pardi, N. Hengartner, P. J. C. Lin, Y. Tam, P. A. Shaw, M. G. Lewis, C. Boesler, U. Şahin, P. Acharya, B. F. Haynes, B. Korber, D. C. Montefiori, D614G Spike Mutation Increases SARS CoV-2 Susceptibility to Neutralization. Cell Host Microbe 29, 23-31.e4 (2021). doi:10.1016/i.chom.2020.11.012 Medline

30. E. J. Anderson, N. G. Rouphael, A. T. Widge, L. A. Jackson, P. C. Roberts, M. Makhene, J. D. Chappell, M. R. Denison, L. J. Stevens, A. J. Pruijssers, A. B. McDermott, B. Flach, B. C. Lin, N. A. Doria-Rose, S. O'Dell, S. D. Schmidt, K. S. Corbett, P. A. Swanson 2nd, M. Padilla, K. M. Neuzil, H. Bennett, B. Leav, M. Makowski, J. Albert, K. Cross, V. V. Edara, K. Floyd, M. S. Suthar, D. R. Martinez, R. Baric, W. Buchanan, C. J. Luke, V. K. Phadke, C. A. Rostad, J. E. Ledgerwood, B. S. Graham, J. H. Beigel, mRNA-1273 Study Group, Safety and Immunogenicity of SARS-CoV-2 mRNA-1273 Vaccine in Older Adults. N. Engl. J. Med. 383, 24272438 (2020). doi:10.1056/NEJMoa2028436 Medline

31. L. Zhang, C. B. Jackson, H. Mou, A. Ojha, H. Peng, B. D. Quinlan, E. S. Rangarajan, A. Pan, A. Vanderheiden, M. S. Suthar, W. Li, T. Izard, C. Rader, M. Farzan, H. Choe, SARS-CoV-2 spike-protein D614G mutation increases virion spike density and infectivity. Nat. Commun. 11, 6013 (2020). doi:10.1038/s41467-020-19808-4 Medline

32. L. Yurkovetskiy, X. Wang, K. E. Pascal, C. Tomkins-Tinch, T. P. Nyalile, Y. Wang, A. Baum, W. E. Diehl, A. Dauphin, C. Carbone, K. Veinotte, S. B. Egri, S. F. Schaffner, J. E. Lemieux, J. B. Munro, A. Rafique, A. Barve, P. C. Sabeti, C. A. Kyratsous, N. V. Dudkina, K. Shen, J. Luban, Structural and Functional Analysis of the D614G SARS-CoV-2 Spike Protein Variant. Cell 183, 739-751.e8 (2020). doi:10.1016/j.cell.2020.09.032

33. T. Zhou, Y. Tsybovsky, J. Gorman, M. Rapp, G. Cerutti, G.-Y. Chuang, P. S. Katsamba, J. M. Sampson, A. Schön, J. Bimela, J. C. Boyington, A. Nazzari, A. S. Olia, W. Shi, M. Sastry, T. Stephens, J. Stuckey, I-T. Teng, P. Wang, S. Wang, B. Zhang, R. A. Friesner, D. D. Ho, J. R. Mascola, L. Shapiro, P. D. Kwong, Cryo-EM Structures of SARS-CoV-2 Spike without and with ACE2 Reveal a pH-Dependent Switch to Mediate Endosomal Positioning of Receptor-Binding Domains. Cell Host Microbe 28, 867-879.e5 (2020). doi:10.1016/j.chom.2020.11.004 Medline

34. X. Xiong, K. Qu, K. A. Ciazynska, M. Hosmillo, A. P. Carter, S. Ebrahimi, Z. Ke, S. H. W. Scheres, L. Bergamaschi, G. L. Grice, Y. Zhang, CITIID-NIHR COVID-19 BioResource Collaboration, J. A. Nathan, S. Baker, L. C. James, H. E. Baxendale, I. Goodfellow, R. Doffinger, J. A. G. Briggs, A thermostable, closed SARS-CoV-2 spike protein trimer. Nat. Struct. Mol. Biol. 27, 934-941 (2020). doi:10.1038/s41594-020-0478-5 Medline

35. T. Xiao, J. Lu, J. Zhang, R. I. Johnson, L. G. A. McKay, N. Storm, C. L. Lavine, H. Peng, Y. Cai, S. Rits-Volloch, S. Lu, B. D. Quinlan, M. Farzan, M. S. Seaman, A. Griffiths, B. Chen, A trimeric human angiotensin-converting enzyme 2 as an antiSARS-CoV-2 agent. Nat. Struct. Mol. Biol. 28, 202-209 (2021). doi:10.1038/s41594-020-00549-3 Medline

36. S. H. Scheres, RELION: Implementation of a Bayesian approach to cryo-EM structure determination. J. Struct. Biol. 180, 519-530 (2012). doi:10.1016/i.jsb.2012.09.006 Medline

37. C. Toelzer, K. Gupta, S. K. N. Yadav, U. Borucu, A. D. Davidson, M. Kavanagh Williamson, D. K. Shoemark, F. Garzoni, O. Staufer, R. Milligan, J. Capin, A. J. Mulholland, J. Spatz, D. Fitzgerald, I. Berger, C. Schaffitzel, Free fatty acid binding pocket in the locked structure of SARS-CoV-2 spike protein. Science 370, 725730 (2020). doi:10.1126/science.abd3255 Medline

38. J. Hansen, A. Baum, K. E. Pascal, V. Russo, S. Giordano, E. Wloga, B. O. Fulton, Y. Yan, K. Koon, K. Patel, K. M. Chung, A. Hermann, E. Ullman, J. Cruz, A. Rafique, T. Huang, J. Fairhurst, C. Libertiny, M. Malbec, W. Y. Lee, R. Welsh, G. Farr, S. Pennington, D. Deshpande, J. Cheng, A. Watty, P. Bouffard, R. Babb, N. Levenkova, C. Chen, B. Zhang, A. Romero Hernandez, K. Saotome, Y. Zhou, M. Franklin, S. Sivapalasingam, D. C. Lye, S. Weston, J. Logue, R. Haupt, M. Frieman, G. Chen, W. Olson, A. J. Murphy, N. Stahl, G. D. Yancopoulos, C. A. Kyratsous, 
Studies in humanized mice and convalescent humans yield a SARS-CoV-2 antibody cocktail. Science 369, 1010-1014 (2020). doi:10.1126/science.abd0827 Medline

39. X. Chi, R. Yan, J. Zhang, G. Zhang, Y. Zhang, M. Hao, Z. Zhang, P. Fan, Y. Dong, Y. Yang, Z. Chen, Y. Guo, J. Zhang, Y. Li, X. Song, Y. Chen, L. Xia, L. Fu, L. Hou, J. Xu, C. Yu, J. Li, Q. Zhou, W. Chen, A neutralizing human antibody binds to the Nterminal domain of the Spike protein of SARS-CoV-2. Science 369, 650-655 (2020). doi:10.1126/science. abc6952 Medline

40. D. J. Benton, A. G. Wrobel, P. Xu, C. Roustan, S. R. Martin, P. B. Rosenthal, J. J. Skehel, S. J. Gamblin, Receptor binding and priming of the spike protein of SARSCoV-2 for membrane fusion. Nature 588, 327-330 (2020). doi:10.1038/s41586020-2772-0 Medline

41. M. M. Shaik, H. Peng, J. Lu, S. Rits-Volloch, C. Xu, M. Liao, B. Chen, Structural basis of coreceptor recognition by HIV-1 envelope spike. Nature 565, 318-323 (2019). doi:10.1038/s41586-018-0804-9 Medline

42. L. Liu, P. Wang, M. S. Nair, J. Yu, M. Rapp, Q. Wang, Y. Luo, J. F.-W. Chan, V. Sahi, A. Figueroa, X. V. Guo, G. Cerutti, J. Bimela, J. Gorman, T. Zhou, Z. Chen, K.-Y. Yuen, P. D. Kwong, J. G. Sodroski, M. T. Yin, Z. Sheng, Y. Huang, L. Shapiro, D. D. Ho, Potent neutralizing antibodies against multiple epitopes on SARS-CoV-2 spike. Nature 584, 450-456 (2020). doi:10.1038/s41586-020-2571-7 Medline

43. K. Wu, A. P. Werner, J. I. Moliva, M. Koch, A. Choi, G. B. E. Stewart-Jones, H. Bennett, S. Boyoglu-Barnum, W. Shi, B. S. Graham, A. Carfi, K. S. Corbett, R. A. Seder, D. K. Edwards, mRNA-1273 vaccine induces neutralizing antibodies against spike mutants from global SARS-CoV-2 variants. bioRxiv 2021.01.25.427948 [Preprint]. 25 January 2021. https://doi.org/10.1101/2021.01.25.427948.

44. C. K. Wibmer, F. Ayres, T. Hermanus, M. Madzivhandila, P. Kgagudi, B. Oosthuysen, B. E. Lambson, T. de Oliveira, M. Vermeulen, K. van der Berg, T. Rossouw, M. Boswell, V. Ueckermann, S. Meiring, A. von Gottberg, C. Cohen, L. Morris, J. N. Bhiman, P. L. Moore, SARS-CoV-2 501Y.V2 escapes neutralization by South African COVID-19 donor plasma. Nat. Med. 10.1038/s41591-021-01285-x (2021). doi:10.1038/s41591-021-01285-x Medline

45. Z. Wang, F. Schmidt, Y. Weisblum, F. Muecksch, C. O. Barnes, S. Finkin, D. Schaefer-Babajew, M. Cipolla, C. Gaebler, J. A. Lieberman, T. Y. Oliveira, Z. Yang, M. E. Abernathy, K. E. Huey-Tubman, A. Hurley, M. Turroja, K. A. West, K. Gordon, K. G. Millard, V. Ramos, J. Da Silva, J. Xu, R. A. Colbert, R. Patel, J. Dizon, C. UnsonO'Brien, I. Shimeliovich, A. Gazumyan, M. Caskey, P. J. Bjorkman, R. Casellas, T. Hatziioannou, P. D. Bieniasz, M. C. Nussenzweig, mRNA vaccine-elicited antibodies to SARS-CoV-2 and circulating variants. Nature 10.1038/s41586-02103324-6 (2021). doi:10.1038/s41586-021-03324-6 Medline

46. P. Wang, M. S. Nair, L. Liu, S. Iketani, Y. Luo, Y. Guo, M. Wang, J. Yu, B. Zhang, P. D. Kwong, B. S. Graham, J. R. Mascola, J. Y. Chang, M. T. Yin, M. Sobieszczyk, C. A. Kyratsous, L. Shapiro, Z. Sheng, Y. Huang, D. D. Ho, Antibody Resistance of SARS-CoV-2 Variants B.1.351 and B.1.1.7. bioRxiv 2021.01.25.428137 [Preprint]. 12 February 2021. https://doi.org/10.1101/2021.01.25.428137.

47. G. Frey, H. Peng, S. Rits-Volloch, M. Morelli, Y. Cheng, B. Chen, A fusionintermediate state of HIV-1 gp41 targeted by broadly neutralizing antibodies. Proc. Natl. Acad. Sci. U.S.A. 105, 3739-3744 (2008). doi:10.1073/pnas.0800255105 Medline

48. J. M. Kovacs, J. P. Nkolola, H. Peng, A. Cheung, J. Perry, C. A. Miller, M. S. Seaman, D. H. Barouch, B. Chen, HIV-1 envelope trimer elicits more potent neutralizing antibody responses than monomeric gp120. Proc. Natl. Acad. Sci. U.S.A. 109, 12111-12116 (2012). doi:10.1073/pnas.1204533109 Medline

49. J. Chen, J. M. Kovacs, H. Peng, S. Rits-Volloch, J. Lu, D. Park, E. Zablowsky, M. S. Seaman, B. Chen, Effect of the cytoplasmic domain on antigenic characteristics of HIV-1 envelope glycoprotein. Science 349, 191-195 (2015). doi:10.1126/science.aaa9804 Medline

50. D. N. Mastronarde, Automated electron microscope tomography using robust prediction of specimen movements. J. Struct. Biol. 152, 36-51 (2005). doi:10.1016/i.jsb.2005.07.007 Medline

51. S. Q. Zheng, E. Palovcak, J.-P. Armache, K. A. Verba, Y. Cheng, D. A. Agard, MotionCor2: Anisotropic correction of beam-induced motion for improved cryoelectron microscopy. Nat. Methods 14, 331-332 (2017). doi:10.1038/nmeth.4193 Medline

52. A. Rohou, N. Grigorieff, CTFFIND4: Fast and accurate defocus estimation from electron micrographs. J. Struct. Biol. 192, 216-221 (2015). doi:10.1016/i.jsb.2015.08.008 Medline

53. P. Emsley, B. Lohkamp, W. G. Scott, K. Cowtan, Features and development of Coot Acta Cryst. D66, 486-501 (2010). doi:10.1107/S0907444910007493 Medline

54. P. D. Adams, P. V. Afonine, G. Bunkóczi, V. B. Chen, I. W. Davis, N. Echols, J. J. Headd, L.-W. Hung, G. J. Kapral, R. W. Grosse-Kunstleve, A. J. McCoy, N. W. Moriarty, R. Oeffner, R. J. Read, D. C. Richardson, J. S. Richardson, T. C. Terwilliger, P. H. Zwart, PHENIX: A comprehensive Python-based system for macromolecular structure solution. Acta Cryst. D66, 213-221 (2010). doi:10.1107/S0907444909052925 Medline

55. T. I. Croll, ISOLDE: A physically realistic environment for model building into lowresolution electron-density maps. Acta Cryst. D74, 519-530 (2018). doi:10.1107/S2059798318002425 Medline

56. A. Morin, B. Eisenbraun, J. Key, P. C. Sanschagrin, M. A. Timony, M. Ottaviano, P. Sliz, Collaboration gets the most out of software. elife 2, e01456 (2013) doi:10.7554/eLife.01456 Medline

\section{ACKNOWLEDGMENTS}

We thank the SBGrid team for technical assistance, K. Arnett for support and advice on the BLI experiments, and S. Harrison, M. Liao, A. Carfi and D. Barouch, A. Burgin and R. Meijers for critical reading of the manuscript. EM data were collected at the Harvard Cryo-EM Center for Structural Biology of Harvard Medical School. We acknowledge support for COVID-19 related structural biology research at Harvard from the Nancy Lurie Marks Family Foundation and the Massachusetts Consortium on Pathogen Readiness (MassCPR). Funding: This work was supported by NIH grants Al147884 (to B.C.), Al147884-01A1S1 (to B.C), Al141002 (to B.C.), Al127193 (to B.C. and James Chou), a COVID-19 Award by MassCPR (to B.C.), as well as a Fast grant by Emergent Ventures (to B.C.). Author contributions: B.C. J.Z., Y.C. and T.X. conceived the project. Y.C. and H.P. expressed and purified the full-length $S$ proteins. T.X. expressed and purified soluble ACE2 constructs with the help from H.P. T.X. performed BLI and cell-cell fusion experiments. J.Z. prepared cryo grids and performed EM data collection with contributions from S.M.S. and R.M.W. J.Z. processed the cryo-EM data, built and refined the atomic models. J.L. created the G614 expression construct. S.R.V. contributed to cell culture and protein production. H.Z., A.N.W. and W.Y. produced antibodies and performed the flow cytometry experiments. P.S. provided computational support. All authors analyzed the data. B.C., J.Z., Y.C. and T.X. wrote the manuscript with input from all other authors. Competing interests: W.Y. serves on the scientific advisory boards of Hummingbird Bioscience and GO Therapeutics and is a consultant to GV20 Oncotherapy. All other authors declare no competing interests. Data and materials availability: The atomic structure coordinates are deposited in the RCSB Protein Data Bank (PDB) under the accession numbers 7KRQ, 7KRR and 7KRS; and the electron microscopy maps have been deposited in the Electron Microscopy Data Bank (EMDB) under the accession numbers EMD-23010, EMD-23011 and EMD-23012. All materials generated during the current study are available from the corresponding author under an MTA with Boston Children's Hospital. This work is licensed under a Creative Commons Attribution 4.0 International (CC BY 4.0) license, which permits unrestricted use, distribution, and reproduction in any medium, provided the original work is properly cited. To view a copy of this license, visit https://creativecommons.org/licenses/by/4.0/. This license does not apply to figures/photos/artwork or other content included in the article that is credited to a third party; obtain authorization from the rights holder before using such material.

\section{SUPPLEMENTARY MATERIALS}

science.sciencemag.org/cgi/content/full/science.abf2303/DC1

Materials and Methods

Figs. S1 to S15

Tables S1 to S3

References (47-56)

MDAR Reproducibility Checklist

12 October 2020; accepted 10 March 2021

Published online 16 March 2021

10.1126/science.abf2303 

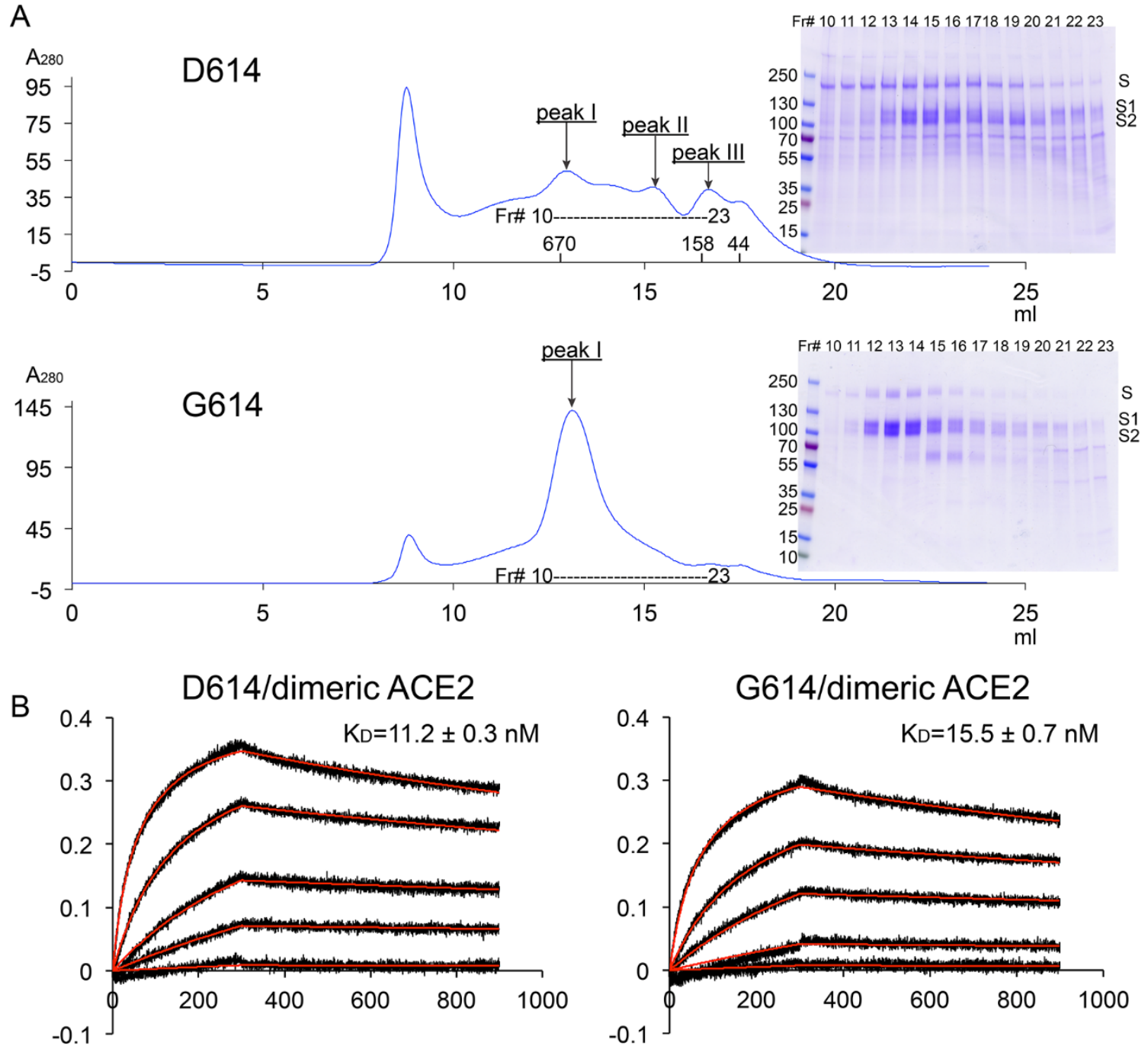

G614/dimeric ACE2
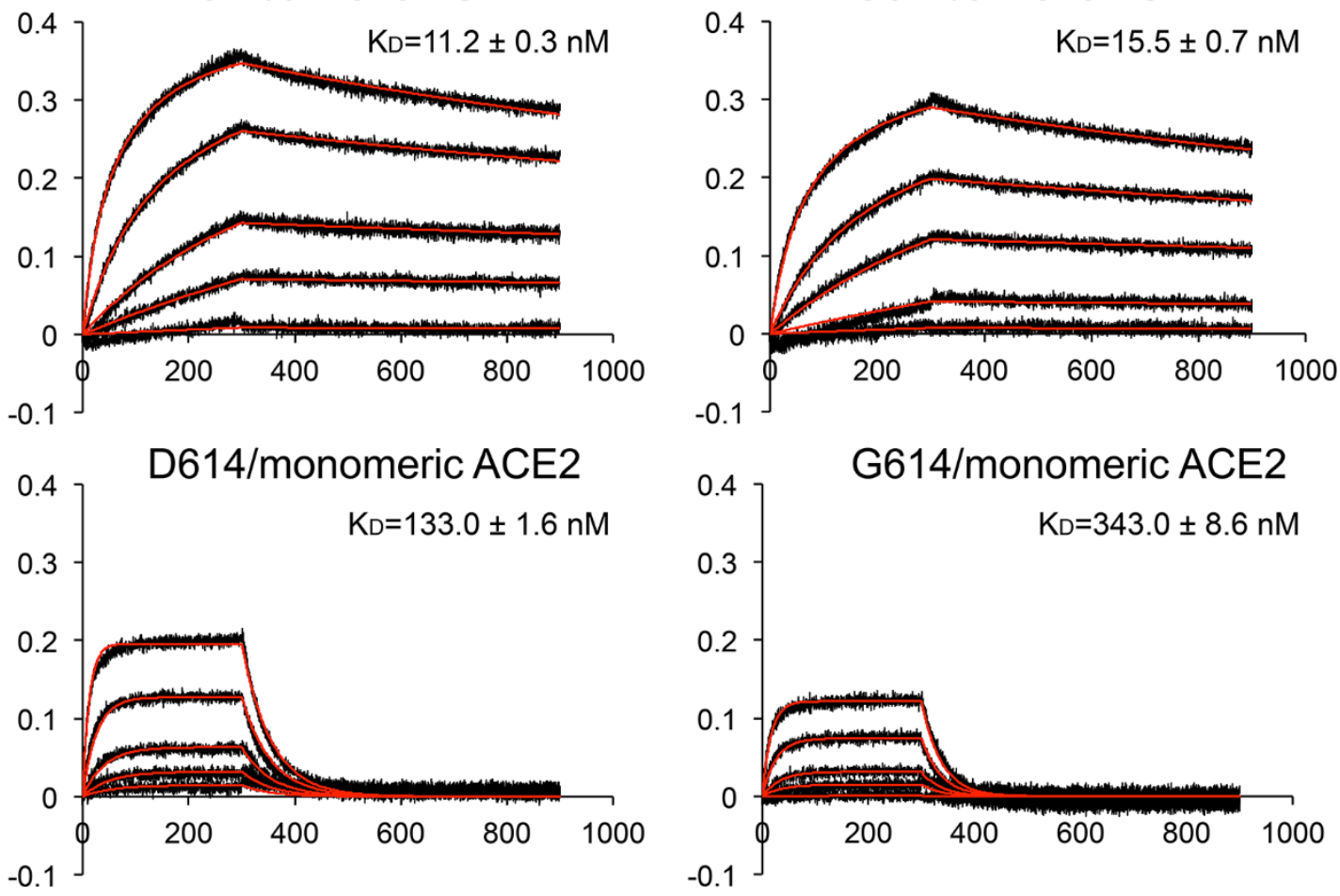

Fig. 1. Characterization of the purified full-length SARS-CoV-2 S proteins. (A) The full-length SARS-CoV-2 S protein carrying either D614 or G614 was extracted and purified in detergent DDM, and further resolved by gelfiltration chromatography on a Superose 6 column. The molecular weight standards include thyoglobulin (670 kDa), $\mathrm{r}$-globulin $(158 \mathrm{kDa})$ and ovalbumin $(44 \mathrm{kDa})$. Peak I, the prefusion S trimer; peak II, the postfusion S2 trimer; and peak III, the dissociated monomeric S1. Inset, peak fractions were analyzed by Coomassie stained SDS-PAGE. Labeled bands are S, S1 and S2. Fr\#, fraction number. (B) Binding analysis of fractions of Peak 1 in (A) with soluble ACE2 constructs by bio-layer interferometry (BLI). The purified S proteins were immobilized to AR2G biosensors and dipped into the wells containing ACE2 at various concentrations (5.56-450 nM for monomeric ACE2, 2.78-225 $\mathrm{nM}$ for dimeric ACE2). Binding kinetics was evaluated using a 1:1 Langmuir binding model for the monomeric ACE2 and a bivalent model for dimeric ACE2. The sensorgrams are in black and the fits in red. Binding constants are also summarized here and in table S1. All experiments were repeated at least twice with essentially identical results. 


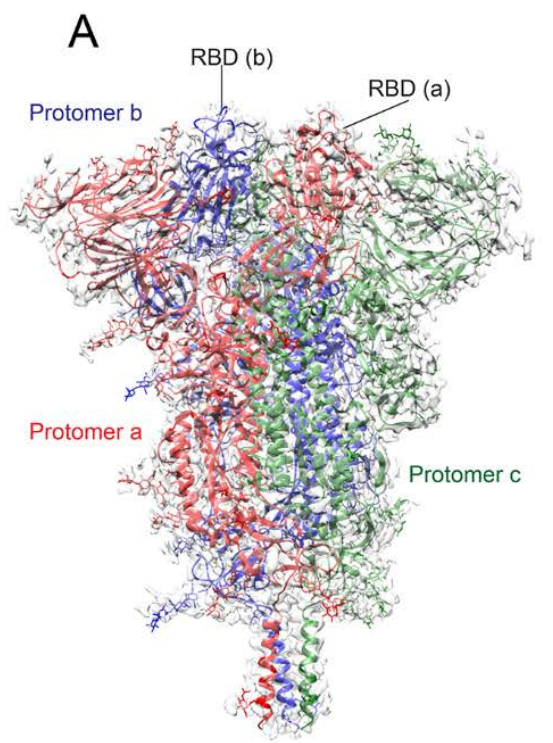

closed conformation

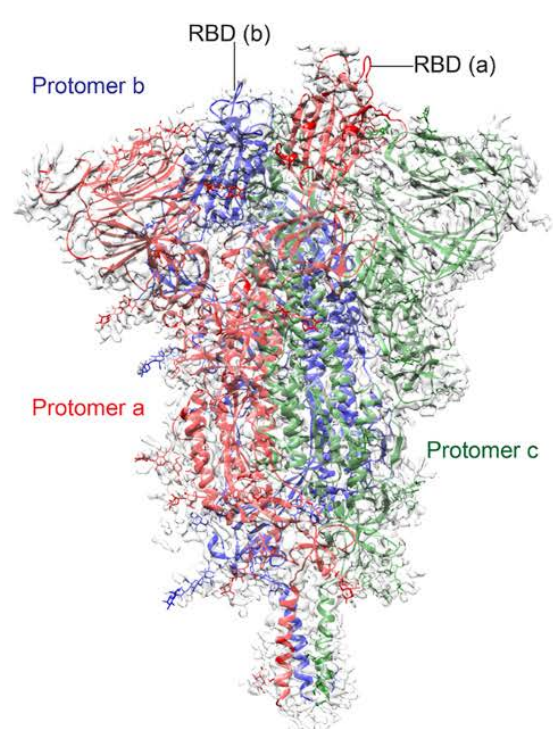

intermediate conformation

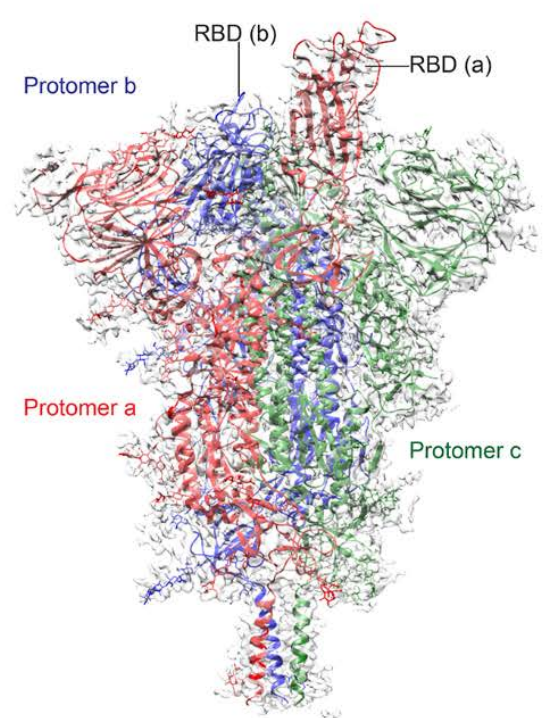

one-RBD-up conformation

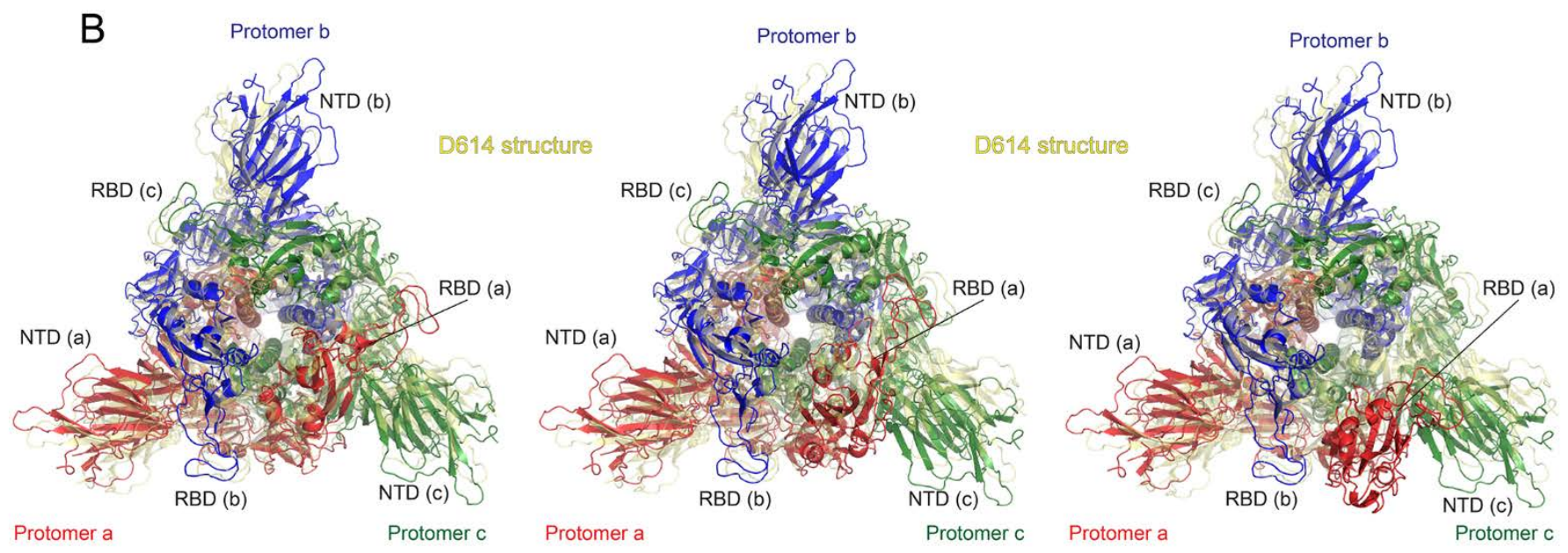

Fig. 2. Cryo-EM structures of the full-length SARS-CoV-2 S protein carrying G614. (A) Three structures of the G614 S trimer, representing a closed, three RBD-down conformation, an RBD-intermediate conformation and a one RBD-up conformation, were modeled based on corresponding cryo-EM density maps at 3.1-3.5 resolution. Three protomers ( $a, b, c)$ are colored in red, blue and green, respectively. RBD locations are indicated. (B) Top views of superposition of three structures of the G614 S in (A) in ribbon representation with the structure of the prefusion trimer of the D614 S (PDB ID: 6XR8), shown in yellow. NTD and RBD of each protomer are indicated. Side views of the superposition are shown in fig. S8. 

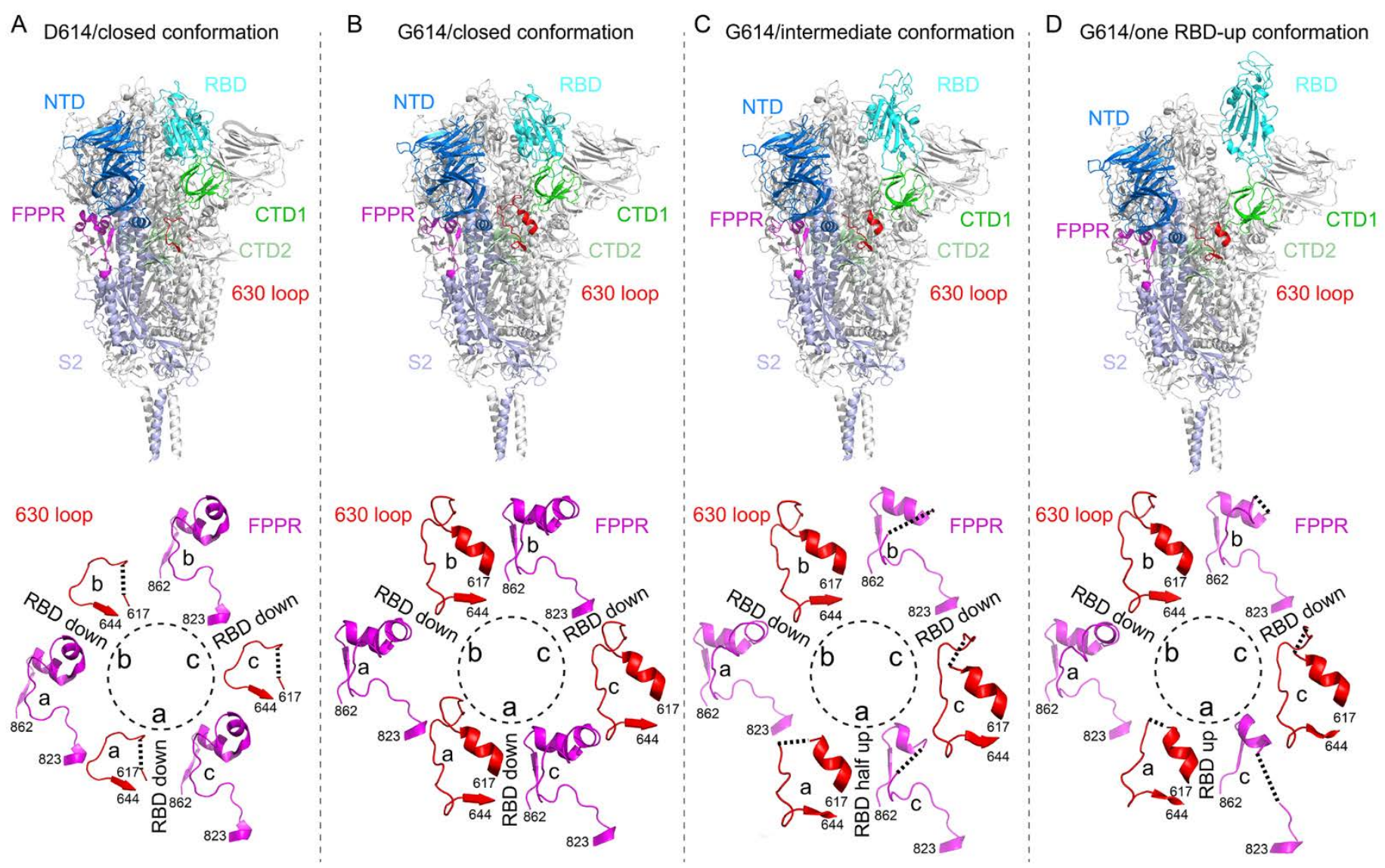

Fig. 3. Cryo-EM structures of the full-length SARS-CoV-2 S protein carrying G614. (A) Top, the structure of the closed, three RBD-down conformation of the D614 S trimer is shown in ribbon diagram with one protomer colored as NTD in blue, RBD in cyan, CTD1 in green, CTD2 in light green, S2 in light blue, the 630 loop in red and the FPPR in magenta. Bottom, structures of three segments (residues 617-644) containing the 630 loop in red and three segments (residues 823-862) containing the FPPR in magenta from all three protomers ( $a, b$ and $c$ ) are shown. Position of each RBD is indicated. ( $B$ to $D$ ) Structures of the G614 trimer in the closed, three RBD-down conformation, the RBD-intermediate conformation and the one RBD-up conformation, respectively, are shown as in (A). Dash lines indicate gaps. 
A

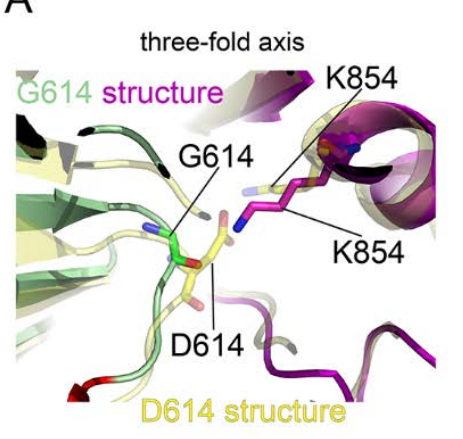

C

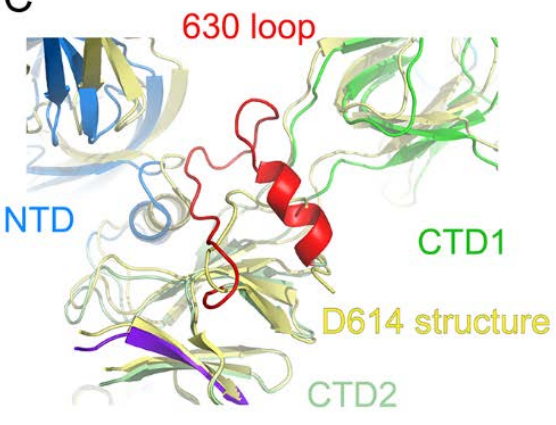

B

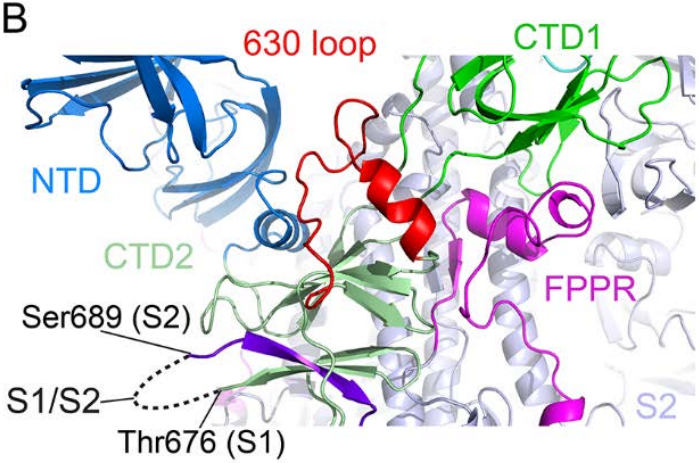

D NTD

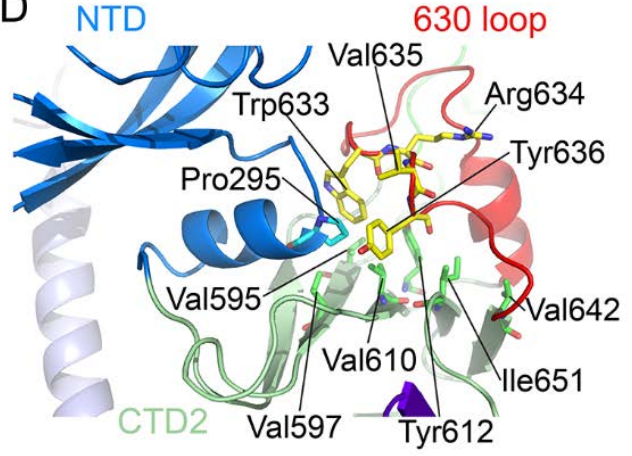

Fig. 4. Close-up views of the D614G substitution. (A) A close-up view of the region near the residue 614 with superposition of the G614 trimer structure in green (CTD2) and magenta (FPPR) and the D614 trimer in yellow, both in the closed prefusion conformation. Residues G614, D614 and two K854 from both structures are shown in stick model. The direction of the three-fold axis of the trimer is indicated. (B) Location of the 630 loop in the $\mathrm{S}$ trimer. The 630 loop is highlighted in red, NTD in blue, CTD1 in green, CTD2 in light green, S2 in light blue, and the FPPR from a neighboring protomer in magenta. The S1/S2 boundary and the nearest ordered residues Thr676 from S1 and S689 from S2 are all indicated. A strand from the $\mathrm{N}$-terminal end of $\mathrm{S} 2$, packed in the CTD2, is highlighted in purple. (C) A view showing that the 630 loop wedges between the NTD and the CTD1 and pushed them apart. (D) Packing of the 630 loop against the hydrophobic surface formed by residues Val595, Val597, Val610 Tyr 612, Val642 and Ile 651 from the CTD2 and Pro295 from the NTD. Residues Trp633 and Val635 from the 630 loop contribute to this interaction. 